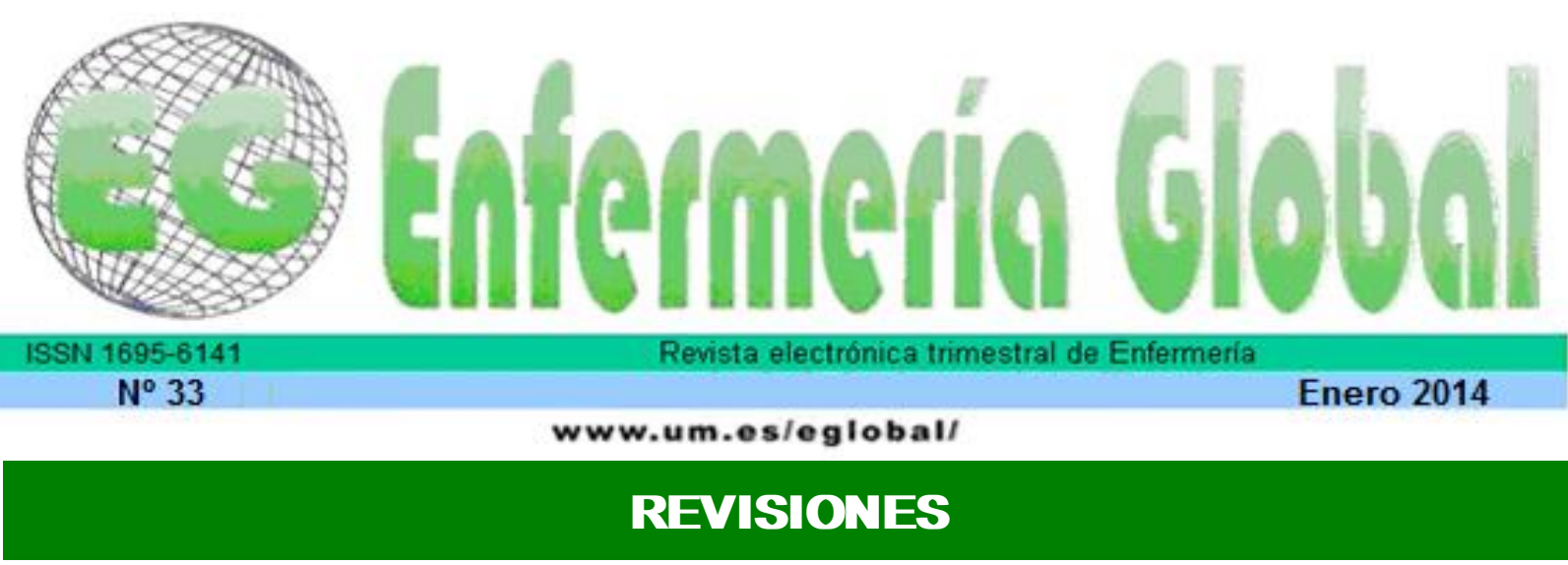

\title{
Errores de medicación en Unidades de Terapia Intensiva Neonatal
}

Erros de medicação em Unidades de Terapia Intensiva Neonatal

Medication errors in Neonatal Intensive Care Units

\section{"Dias da Silva, Gustavo ${ }^{* *}$ Silvino, Zenith Rosa ***Saraiva de Almeida, Viviane ${ }^{* * * *}$ Lemos Querido, Danielle ${ }^{* * * * *}$ Silva Cunha Dias, Pollyanna ******Pompeu Christovam, Bárbara}

*Miembro del Núcleo de Estudios e Investigaciones en Ciudadanía y Gerencia en Enfermería (NECIGEN). Director de Enfermería de la Maternidade Escola de la UFRJ. E-mail: gustavodias@me.ufrj.br **Doctora enm Enfermería. Titular del Departamento de Fundamentos de Enfermería y Administración de la Escuela de Enfermería Aurora de Afonso Costa (EEAAC/UFF). Coordinadora del NECIGEN. Niterói (RJ) *** Vicediretora de Enfermería de la Maternidade-Escola de la UFRJ. ${ }^{* * * *}$ Coordinadora de Enfermería de la Unidad Neonatal de la Maternidade-Escola de la UFRJ. ${ }^{* * * * *}$ Enfermera. Especialista en Terapia Intensiva y Cardiología del Hospital Federal de los Servidores del Estado (HFSE/MS). Rio de Janeiro. ${ }^{* * * * * *}$ Doctora en Enfermería. Profesora Adjunta del Departamento de Fundamentos de Enfermería y Administración de la Escuela de Enfermería Aurora de Afonso Costa (EEAAC/UFF). Niterói (RJ), Brasil.

Palabras clave: Errores de medicación; Unidades de Cuidado Intensivo Neonatal; Enfermería; Administración de la Seguridad; Calidad de la Atención de Salud

Palavras-chave: Erros de medicação; UTI Neonatal; Enfermagem; Gerenciamento de Segurança; Qualidade da Assistência à Saúde.

Keywords: Medication errors; Neonatal Intensive Care Units; Nursing; Safety Management; Quality of Health Care.

\section{RESUMEN}

Se trata de una revisión integradora de la literatura realizada en bases de datos en línea en el período 2002-2012, con el objetivo de identificar la evidencia disponible sobre la incidencia, los factores relacionados, las consecuencias y los mecanismos para la prevención de errores de medicación en la Unidad de Cuidados Intensivos Neonatales. Los resultados se clasifican de acuerdo a los temas centrales de la revisión del diseño. Llegamos a la conclusión de que un conocimiento más profundo de la magnitud de los errores y eventos adversos de medicamentos en la Unidad de Cuidados Intensivos 
Neonatales, su impacto en la morbilidad y mortalidad neonatal, factores asociados con este fenómeno y los mecanismos de prevención son las estrategias para la construcción de un sistema de salud más seguro y de calidad.

\section{RESUMO}

Trata-se de uma revisão integrativa da literatura realizada em bases de dados online, no período entre 2002-2012, com o objetivo de identificar as publicações disponíveis sobre incidência, fatores relacionados, consequências e/ou mecanismos de prevenção dos erros de medicação na Unidade de Tratamento Intensivo Neonatal. Os resultados foram categorizados segundo as temáticas centrais do delineamento da revisão. Conclui-se que o conhecimento aprofundado da magnitude dos erros e eventos adversos à medicamentos na Unidade de Tratamento Intensivo Neonatal, seu impacto na morbimortalidade neonatal, os fatores associados a essa ocorrência e os mecanismos de prevenção são estratégias para a constru

\section{ABSTRACT}

This is an integrative literature review conducted in online databases in the period 2002-2012, aiming to identify the available evidence about incidence, related factors, consequences and mechanisms for prevention of medication errors in the unit neonatal intensive care unit. results were categorized according to the central themes of the design review. we conclude that a deeper knowledge of the errors and adverse drug events in the unit neonatal intensive care unit, its impact on neonatal morbidity and mortality, factors associated with this occurrence and prevention mechanisms are strategies for building a safer health system and quality.

\section{INTRODUCCIÓN}

Con el desarrollo cada vez mayor de la ciencia y de la tecnología a ella asociada, sumado a la sofisticación de las unidades de terapia intensiva neonatales y de los recursos terapéuticos, hubo un considerable aumento en la tasa de supervivencia de los recién nacidos gravemente enfermos, especialmente de los bebes prematuros ${ }^{(1)}$.

Acompañando el incremento tecnológico en la asistencia neonatal, los progresos de la terapéutica medicamentosa también han sido notables, influyendo fuertemente en la reducción de la morbilidad y mortalidad. Los medicamentos se han convertido en elementos de primer orden que constituyen herramientas poderosas para mitigar el sufrimiento humano, posibilitando curas, prolongando la vida e impidiendo o retardando la aparición de complicaciones provenientes de las enfermedades. Además, es posible considerar el uso apropiado e inteligente de los medicamentos como tecnología altamente costo-efectiva, una vez que puede influir, de modo sustantivo, en la utilización del resto del cuidado. Con todo, su uso no está exento de riesgo y también puede aumentar los costos de la atención a la salud si utilizados inadecuadamente ${ }^{(2-3)}$.

En medio de los avances tecnológicos y científicos ocurridos en la atención a la salud, principalmente en las últimas décadas, el sistema actual tiene como gran desafío, la prestación de un cuidado seguro, efectivo, oportuno e individualizado, en contextos clínicos y normativamente cada vez más complejos ${ }^{(4)}$.

Según un informe publicado en el año 2000 por el Institute of Medicine (EUA), referencia para muchos estudios, intitulado To error is human: building a safer health system, errores durante la atención prestada a la salud ocurren en cerca de $14 \%$ de los pacientes internados en los EUA. Los autores estimaron cerca de 44.000 a 98.000 muertes anuales debido a problemas causados por errores en la atención a la salud, 
lo que supera a las muertes por vehículos motorizados, cáncer de mama y SIDA. Los errores de medicación fueron responsables de la muerte de aproximadamente 7.000 personas al año ${ }^{(5)}$.

En Brasil la preocupación por la seguridad del paciente y la prevención de errores y eventos adversos también han tomado proporciones cada vez mayores ${ }^{(6)}$.

Los errores que afectan a los medicamentos son clasificados como eventos prevenibles, pudiendo o no resultar en daños a los pacientes. La ocurrencia del daño caracteriza el evento adverso al medicamento, que se refiere al prejuicio o lesión, temporaria o permanente, derivada del uso incorrecto del medicamento, incluyendo la falta del mismo ${ }^{(3-4)}$.

Debido a las peculiaridades de la clientela neonatal, especialmente de los recién nacidos gravemente enfermos, la ocurrencia de errores terapéuticos es muy grande, principalmente de terapia medicamentosa. Eso se debe al hecho de que, por estar en situación de riesgo, ellos demandan la aplicación de alta tecnología, junto con la necesidad de prescripción médica individualizada, basada en peso corporal y edad gestacional $^{(7)}$.

En este contexto, ese estudio objetiva identificar en la literatura los trabajos que tratan de la incidencia, factores relacionados, consecuencias y/o mecanismos de prevención de los errores de medicación en Unidades de Terapia Intensiva Neonatal (UTIN).

\section{METODOLOGÍA}

Para el alcance del objetivo del estudio, optamos por realizar una revisión integrativa de la literatura. La revisión integrativa incluye los análisis de investigación relevantes que dan soporte a la toma de decisión y la mejoría de la práctica clínica, posibilitando la síntesis del estado del conocimiento de un determinado asunto, además de apuntar lagunas del conocimiento que precisan ser completadas con la realización de nuevos estudios $^{(8)}$. Ese método surge como una metodología que proporciona la incorporación de la aplicabilidad de resultados de estudios significativos en la práctica, con propósito de definir conceptos, revisar teorías y evidencias y analizar problemas metodológicos de un tópico peculiar ${ }^{(9)}$.

Aunque los métodos para la conducción de revisiones integrativas varíen, en la realización de esta revisión utilizamos las siguientes etapas: establecimiento de la pregunta de la investigación, selección de la muestra, colecta de datos, análisis y categorización de los estudios, discusión de los resultados y presentación de la revisión.

La presente revisión tuvo como guía la siguiente pregunta: “¿Cuáles son las publicaciones científicas disponibles en las bases de datos sobre la ocurrencia, factores relacionados, consecuencias y/o mecanismos de prevención de los errores de medicación en UTIN?".

La colecta de las publicaciones se realizó en el mes de abril de 2012 en las siguientes bases de datos: PubMed-MEDLINE, SciELO, LILACS, Bases de Datos en Enfermería (BDENF), Biblioteca Digital Brasilera de Tesis y Disertaciones (BDTD) y en el portal de la Coordinación de Perfeccionamiento de Personal de Enseñanza Superior (CAPES). 
Se establecieron como criterios para la selección de la muestra, estudios publicados con texto disponible en la íntegra online en idioma Portugués, Inglés, Español o Francés, publicados entre enero de 2002 y enero 2012, con los descriptores "Errores de medicación" y "UTI Neonatal", elegidos después de la consulta en los Descriptores en Ciencias de la Salud (DeCS), combinados a través del conector booleano "AND". Fueron seleccionados artículos de revisión sistemática, ensayos clínicos aleatoriamente controlados, revisión integrativa, estudio observacional, investigación cualitativa y editorial.

Para la colecta de datos de los artículos que fueron incluidos en la revisión integrativa, se utilizó un instrumento propio usado por investigadores docentes y discentes de un programa de post-graduación en enfermería, adaptado del protocolo de revisión de Polit, Beck y Hungler ${ }^{(10)}$.

Después de la traducción y/o lectura, los artículos fueron analizados, según sus contenidos, por medio de la estadística descriptiva y a la relación de las informaciones con el objeto de interés en cada estudio. Posteriormente los artículos fueron categorizados y los datos extraídos según las temáticas centrales del delineamiento de la revisión, incluyendo la incidencia, etiología, consecuencias y mecanismos de prevención de los errores de medicación en UTIN.

\section{RESULTADOS}

Inicialmente, al procesarse los descriptores combinados por medio de la BVS, se obtuvieron 73 resúmenes. En la investigación de estos descriptores en inglés (MeSH terms) por el PubMed, 36 resúmenes fueron capturados, conformando un total de 109 trabajos. No fue capturado ningún artículo con los descriptores combinados en las otras fuentes de búsqueda.

De los 73 resúmenes encontrados en la BVS, refinamos 7 textos completos disponibles dentro de los criterios de investigación, siendo 6 artículos (1 en la base de datos LILACS y 5 en la base de datos MEDLINE) y 01 disertación (LILACS). De los textos capturados en el PubMed, 10 fueron seleccionados de acuerdo con los criterios pre-establecidos, todos indexados en la base de datos MEDLINE.

Los resultados encontrados por los dos autores que realizaron la búsqueda, dentro de los criterios de elegibilidad, fueron idénticos.

Después de la lectura previa del material seleccionado, en consenso, fueron excluidos 03 artículos del PubMed por no responder a las preguntas que guiaron la búsqueda de los artículos y otros 03 artículos por estar en duplicidad, totalizando para análisis 10 artículos y 01 disertación de maestría. La disertación de maestría (LILACS) también fue excluida, después de la lectura, por el hecho de los resultados haber sido publicados en la íntegra en uno de los artículos seleccionados.

Durante la lectura previa de los resúmenes de los trabajos, un artículo (MEDLINE), que no había sido encontrado por la búsqueda con los descriptores seleccionados, fue incluido en la revisión porque complementa uno de los artículos seleccionados y por tratarse de una extensión de la investigación de este autor, publicado en otra revista con descriptores diferentes (Figura 1). 
Figura 1. Diagrama de flujo de la Revisión.

\begin{tabular}{|l|}
\multicolumn{1}{|c|}{ Box 1} \\
Descriptores (DeCS): \\
\hline 1- Errores de Medicación \\
y \\
2- UTI Neonatal \\
Sistemas de Búsqueda: \\
\hline 1- BVS \\
2- Portal CAPES \\
3- BDTD \\
Bases de Datos: \\
1- LILACS \\
2- MEDLINE \\
3- SCIELO \\
4- BDENF \\
\hline
\end{tabular}

\begin{tabular}{|l|}
\multicolumn{1}{c|}{ Box 2} \\
MeSH terms: \\
\hline 1- Errores de Medicación \\
y \\
2- NICU \\
Sistema de Búsqueda: \\
\hline $1-$ NCI/PubMed \\
Base de Datos: \\
\hline $1-$ MEDLINE \\
\hline
\end{tabular}

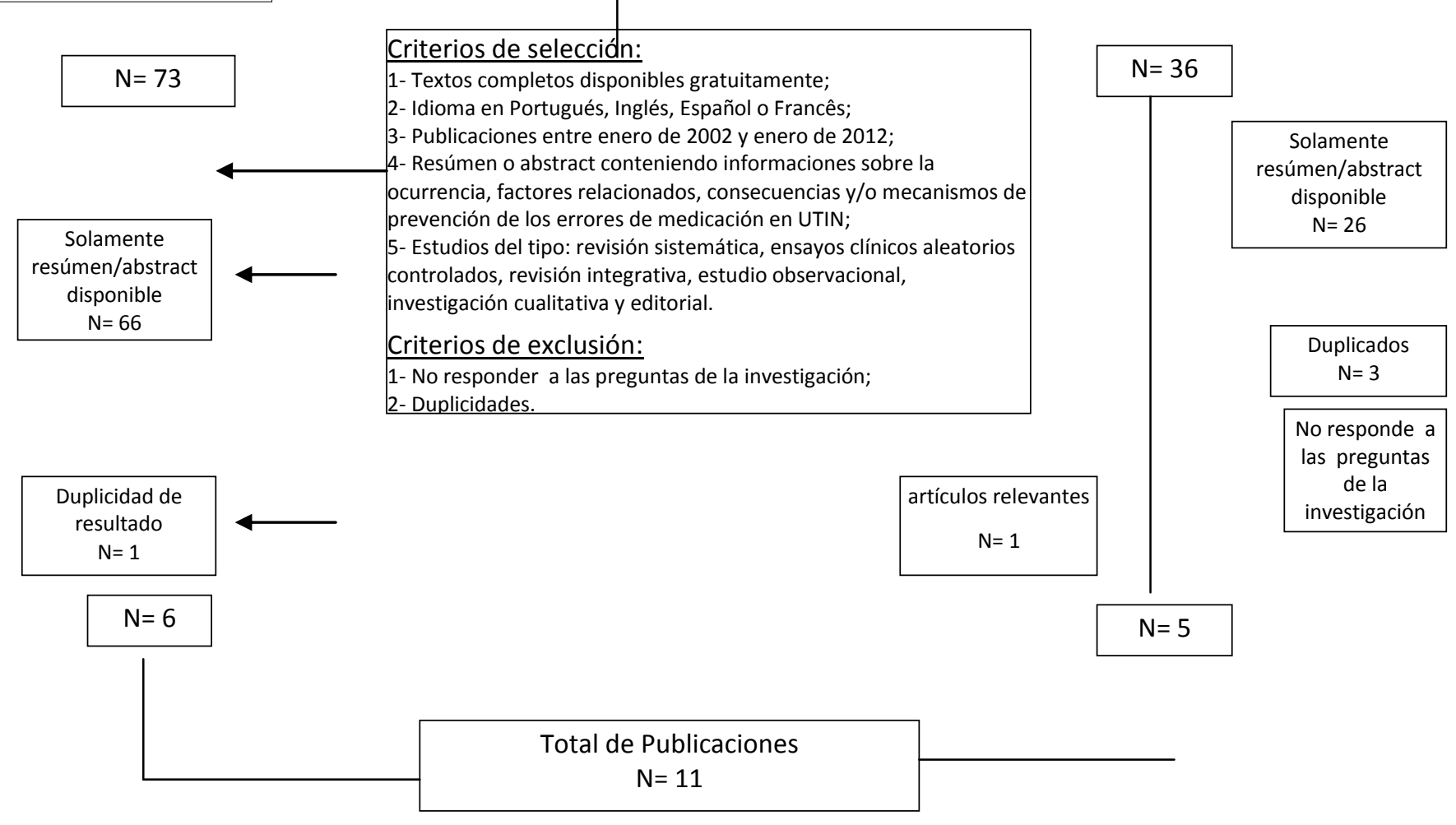

De los 11 estudios seleccionados, $10(90,9 \%)$ eran internacionales y $01(9,1 \%)$ desarrollado en Brasil. De los estudios internacionales, 04 eran norteamericanos, 01 británico, 01 holandés, 01 argentino y 01 indio.

En relación al perfil de los autores de los artículos, su gran mayoría estaba compuesta por médicos $(77,8 \%)$, seguidos de enfermeros $(13,3 \%)$ y farmacéuticos $(8,9 \%)$. Los periódicos donde los artículos refinados fueron publicados eran todos de medicina.

De los artículos seleccionados, 09 (81,8\%) eran primarios y $02 \quad(18,2 \%)$ investigaciones de revisión bibliográfica, siendo una sistemática y un editorial. Todas las investigaciones primarias fueron desarrolladas en campo en la UTIN con aplicación de métodos cuantitativos de investigación, siendo 08 (88,9\%) en hospitales universitarios o vinculados a institutos de investigación, ciencia y tecnología.

De los estudios primarios presentados en la revisión, 7 (77,8\%) tenían diseño 
metodológico prospectivo, de estos 6 fueron desarrollados en dos fases (preintervención y post-intervención), y apenas $2(22,2 \%)$ eran retrospectivos.

Figura 2. Caracterización de la muestra según los objetivos delineados, tipo de estudio y fuentes de datos, 2002-2012.

\begin{tabular}{|c|c|c|c|c|c|}
\hline Objetivos & $\mathbf{N}$ & Tipo de Estudio & $\mathbf{N}$ & Fuente de los Datos & $\mathbf{N}$ \\
\hline $\begin{array}{c}\text { Describir } \\
\text { Incidencia/prevalencia } \\
\text { de errores }\end{array}$ & 8 & Estudios de Revisión & 2 & Prontuario & 9 \\
\hline $\begin{array}{l}\text { Describir los tipos de } \\
\text { errores }\end{array}$ & 3 & Estudios Primarios & 9 & Literatura Científica & 2 \\
\hline $\begin{array}{l}\text { Caracterizar el sistema } \\
\text { de notificaciones de } \\
\text { errores }\end{array}$ & 1 & Retrospectivo & 4 & $\begin{array}{c}\text { Entrevista con } \\
\text { profesionales de salud }\end{array}$ & 2 \\
\hline $\begin{array}{c}\text { Describir la etiología } \\
\text { de los errores }\end{array}$ & 2 & Prospectivo & 1 & $\begin{array}{l}\text { Observación no- } \\
\text { participante }\end{array}$ & 2 \\
\hline $\begin{array}{c}\text { Desarrollar o describir } \\
\text { mecanismos de } \\
\text { prevención de los } \\
\text { errores }\end{array}$ & 8 & $\begin{array}{c}\text { Prospectivo } \\
\text { Longitudinal/Trans } \\
\text { versal }\end{array}$ & 6 & $\begin{array}{c}\text { Informes de } \\
\text { notificaciones voluntaria } \\
\text { de errores }\end{array}$ & 1 \\
\hline
\end{tabular}

En relación a las preguntas abordadas por los artículos seleccionados, considerando el carácter multidimensional de los estudios y que en algunos casos los resultados eran abarcadores y mixtos, 7 trabajos resultaron en la descripción de la ocurrencia, incidencia o prevalencia de los errores de medicación en UTIN.

Figura 3. Identificación de los artículos y análisis según la ocurrencia y factores relacionados de los errores de medicación en UTIN, 2002-2012.

\begin{tabular}{|c|c|c|}
\hline $\begin{array}{l}\text { Categoría } \\
\text { de Análisis }\end{array}$ & Referencia & Resultados \\
\hline \multirow{5}{*}{ Ocurrencia } & $\begin{array}{l}\text { Lerner } \\
\mathrm{RB}^{(11)}\end{array}$ & $\begin{array}{l}\text { - } 55 \% \text { de los prontuarios tenían errores, } 84,2 \% \text { de los errores } \\
\text { eran de medicación. Error más común fue el de programación } \\
\text { incorrecta de la bomba de infusión }(88 \%) \text {. }\end{array}$ \\
\hline & $\begin{array}{l}\text { Snijders } \\
\mathrm{C}^{(12)}\end{array}$ & $\begin{array}{l}\text { - } 7 \text { estudios de errores de medicación, relato más frecuente en } \\
\text { todos los estudios; Errores más comunes fueron de } \\
\text { prescripción errada o administración errada. }\end{array}$ \\
\hline & & $\begin{array}{l}\text { - } 105 \text { errores, } 4 \text { graves, } 45 \text { potencialmente graves y } 56 \text { de } \\
\text { menor importancia; }\end{array}$ \\
\hline & $\begin{array}{l}\text { Simpson } \\
\mathrm{JH}^{(13)}\end{array}$ & $\begin{array}{l}\text { - } 60 \% \text { asociados a las medicaciones parenterales, siendo la } \\
\text { gentamicina el medicamento más envuelto }(33 \%) \text {, seguido de } \\
\text { penicilina cristalina (16\%) y vancomicina }(14 \%) \text {; } \\
\text { - } 29 \% \text { de los errores eran de administración; } \\
\text { - Errores de medicación son los más comunes en UTIN. }\end{array}$ \\
\hline & Gray $\mathrm{JE}^{(14)}$ & $\begin{array}{l}\text { Eventos adversos a medicamentos pueden ocurrir hasta ocho } \\
\text { veces más frecuentemente en UTI Neonatal de que en } \\
\text { pacientes adultos; }\end{array}$ \\
\hline
\end{tabular}




\begin{tabular}{|c|c|c|}
\hline & $\begin{array}{l}\text { Walsh } \\
\mathrm{KE}^{(16)} \\
\text { Otero } \mathrm{P}^{(17)}\end{array}$ & $\begin{array}{l}\text { - 9,6\% de las prescripciones contenían errores, taza de errores } \\
\text { de } 1,5 \text { y } 0,7 / \text { pacientes en el DE y UTIN, respectivamente, } \\
\text { siendo significativamente mayores en el } \mathrm{DE}(\mathrm{P}<0,001) \text {; } \\
\text { - Los errores más comunes fueron de dosificación, la mayoría } \\
\text { de los errores eran evitables. } \\
\text { - Errores de dosificación fueron los más comunes, seguidos } \\
\text { por los errores de administración. } \\
\text { - Errores más frecuentes en ambas fases fueron de } \\
\text { administración. }\end{array}$ \\
\hline $\begin{array}{l}\text { Factores } \\
\text { Relaciona } \\
\text { dos }\end{array}$ & $\begin{array}{l}\text { Chappell } \\
\mathrm{K}^{(18)} \\
\text { Simpson } \\
\mathrm{JH}^{(13)} \\
\text { Gray JE }{ }^{(14)}\end{array}$ & $\begin{array}{l}\text { - Ocurrencia de errores significativamente mayor durante el } \\
\text { día (64\% versus } 34 \%) \text {; } \\
\text { - No hubo correlación significativa entre errores y la razón } \\
\text { paciente/enfermera. } \\
\text { - Tasas de errores mayores en estudios de notificación } \\
\text { voluntaria ( } 13-14,7 \text { por } 1000 \text { pacientes de UTI/día versus } 0,97 \\
\text { por } 1000 \text { pacientes de UTI/día; } \\
\text { - Etiología: no observancia a los procedimientos } \\
\text { estandarizados, desatención, documentación escasa o falla de } \\
\text { comunicación. } \\
\text { - } 31 \% \text { de prescripciones de dosis inferiores a } 1 / 10 \text { del frasco, } \\
4,8 \% \text { con dosis inferiores a } 1 / 100 \text { del frasco. } \\
\text { - } 71 \% \text { de los errores estaban asociados a las prescripciones } \\
\text { con pocas informaciones. } \\
\text { - Dosis muy fraccionadas, variaciones de cálculo, falta de } \\
\text { estandarización de dosis, tubos de alimentación enteral y } \\
\text { líneas endovenosas con conexiones semejantes. } \\
\text { - Edad <2 meses (OR } 6,43) \text {, horario nocturno (OR } 3,06) \text {, sin } \\
\text { diferencia significativa entre días de semana y finales de } \\
\text { semana en las dos fases. } \\
\text { - Sin diferencia en las tasas de errores entre los horarios } \\
\text { analizados. } \\
\text { - Errores de administración (N=22), problemas de } \\
\text { prescripción (N=20) y problemas en la farmacia (N=18) } \\
\text { fueron responsables por } 74 \% \text { del total de errores. }\end{array}$ \\
\hline
\end{tabular}

En 8 de los artículos, encontramos en los resultados posibles factores relacionados o no relacionados al error, o también situaciones o características que pueden 0 no aumentar la chance del error. Las estrategias de prevención de los errores fueron pormenorizadas en 8 estudios, y apenas 5 de los 11 artículos filtrados apuntaban una o más consecuencias de los errores de medicación para los recién nacidos internados en la UTIN, siendo que de estos 2 estudios eran de revisión y ninguno de ellos objetivó directamente evaluar los resultados de estos eventos y el impacto en la morbilidad y mortalidad neonatal. 
Figura 4. Identificación de los artículos y análisis según las consecuencias y mecanismos de prevención de los errores de medicación en UTIN, 2002-2012.

\begin{tabular}{|c|c|c|}
\hline $\begin{array}{l}\text { Categoría } \\
\text { de Análisis }\end{array}$ & Referencia & Resultados \\
\hline \multirow{5}{*}{$\begin{array}{l}\text { Consecuen } \\
\text { cias }\end{array}$} & $\begin{array}{l}\text { Snijders } \\
\mathrm{C}^{(12)}\end{array}$ & $\begin{array}{l}\text { - Un estudio con relato de daño real. Incidentes } \\
\text { potencialmente perjudiciales fueron relatados en casi todos los } \\
\text { estudios. Un estudio relató la necesidad de intervención } \\
\text { terapéutica específica en } 30 \% \text { de los casos. }\end{array}$ \\
\hline & $\begin{array}{l}\text { Simpson } \\
\mathrm{JH}^{(13)}\end{array}$ & $\begin{array}{l}\text { - } 4 \text { errores graves, } 2 \text { por sobredosis, una de ellas necesitando } \\
\text { de antagonista. }\end{array}$ \\
\hline & Otero $\mathrm{P}^{(17)}$ & - Ningún evento adverso fue identificado en el estudio. \\
\hline & Jain $S^{(15)}$ & $\begin{array}{l}\text { - Errores sin daños significativos, algunos con potencial para } \\
\text { complicaciones }(11 \%) \text {. }\end{array}$ \\
\hline & $\begin{array}{l}\text { Walsh } \\
\mathrm{KE}^{(16)}\end{array}$ & - 23 errores resultaron en lesión (7/1000 pacientes-día). \\
\hline \multirow{7}{*}{$\begin{array}{l}\text { Mecanismos } \\
\text { de } \\
\text { Prevención }\end{array}$} & $\begin{array}{l}\text { Snijders } \\
\mathrm{C}^{(12)}\end{array}$ & $\begin{array}{l}\text { - Sistemas de prescripción estandarizada e informatizada, } \\
\text { farmacias satélites, farmacéutico clínico, duplo chequeo. }\end{array}$ \\
\hline & $\begin{array}{l}\text { Simpson } \\
\mathrm{JH}^{(13)}\end{array}$ & $\begin{array}{l}\text { - El programa de gerenciamiento de riesgos, educación } \\
\text { permanente, reducción de errores de } 24,1 \text { por } 1000 \\
\text { pacientes/día para } 5,1 \text { por } 1000 \text { pacientes/día }(\mathrm{p}<0,001) \text {. } \\
\text { - Trabajo multidisciplinar, "cultura de la seguridad" del } \\
\text { paciente, profesional y ambiente, "cinco ciertos", } \\
\text { farmacéuticos clínicos, prescripción médica informatizada y } \\
\text { mejor comunicación. }\end{array}$ \\
\hline & $\begin{array}{l}\text { Lehmann } \\
\mathrm{CU}^{(20)}\end{array}$ & $\begin{array}{l}\text { - La implantación de un sistema informatizado de pedidos de } \\
\text { Nutrición Parenteral Total (TPNCalculator) redujo } 61 \% \text { de los } \\
\text { errores de prescripción de NPT (10,8 errores/100 }\end{array}$ \\
\hline & $\begin{array}{l}\text { Lehmann } \\
\mathrm{CU}^{(21)}\end{array}$ & $\begin{array}{l}\text { prescripciones para } 4,2 \text { errores } / 100 \text { prescripciones }-\mathrm{p}<0,01) \text {. } \\
\text { - Después de TPNCalculator, hubo reducción de } 89 \% \text { en las } \\
\text { tasas de errores ( } 10,8 \text { errores } / 100 \text { prescripciones para } 1,2 \\
\text { errores/100 prescripciones). }\end{array}$ \\
\hline & Otero $\mathrm{P}^{(17)}$ & $\begin{array}{l}\text { - Cultura de seguridad positiva, gestión no-punitiva, } \\
\text { farmacéuticos e instrumentos tipo checklist redujeron } \\
\text { significativamente la prevalencia de errores }(7,3 \% \text { x } 11,4 \% \text {, } \\
\text { diferencia de riesgo de } 4,1 \% \text {; }<<0,05 \text {; OR: } 0,61) \text {. }\end{array}$ \\
\hline & $\begin{array}{l}\text { Walsh } \\
\mathrm{KE}^{(16)}\end{array}$ & $\begin{array}{l}\text { - Prescripción computadorizada redujo la tasa de errores de } 38 \\
\text { por } 1000 \text { pacientes-día para } 8 \text { en cada } 1000 \text { pacientes-día. } \\
\text { - Prescripción computarizada redujo errores de administración }\end{array}$ \\
\hline & $\begin{array}{l}\text { Taylor } \\
\mathrm{JA}^{(19)}\end{array}$ & $\begin{array}{l}\text { y problemas de prescripción redujeron (RR } 0,45 \text { X RR } 0,56) \text { ). } \\
\text { La administración del medicamento redujo de } 9,9 \% \text { para } \\
6,7 \% \text {, seguida de dosis errada de } 4,3 \% \text { para } 3 \% \text { y vía errada } \\
\text { de } 4 \% \text { para } 0 \% \text {. }\end{array}$ \\
\hline
\end{tabular}

\section{DISCUSIÓN}

Se verifica que la disponibilidad de la producción científica que objetiva identificar, comprender y buscar soluciones para prevención de los errores de medicación en UTI Neonatal todavía es incipiente. En contrapartida, a causa de las peculiaridades de 
esta clientela, la susceptibilidad a los errores y eventos adversos es mucho mayor en comparación con los pacientes adultos ${ }^{(4,14)}$.

En especial, se nota la carencia de estudios nacionales, ya que solo un estudio brasileño $^{(11)}$ fue seleccionado por los criterios de muestra. También podemos inferir que la producción científica con este foco todavía está restringida a las instituciones de atención a la salud vinculadas a las universidades o institutos de investigación, ya que solo un estudio primario ${ }^{(13)}$ fue desarrollado en un hospital sin este vínculo aparente.

A pesar de la pluralidad del tema e inespecíficas del mismo, se percibe que el interés en estudiar las preguntas en relación a los errores de medicación y sus interfaces con la atención neonatal, todavía se concentra más en los profesionales médicos.

El sistema de medicaciones es complejo y compuesto por varios procesos, pudiendo variar de 20 a 60 etapas diferentes involucradas en los procesos de prescripción, dispensación y administración de medicamentos. El abarcar a muchos profesionales da a ese ciclo la característica multidisciplinar y genera múltiples transferencias de pedidos o materias, que pueden favorecer la ocurrencia de errores ${ }^{(22)}$.

La complejidad que envuelve la práctica de la terapia medicamentosa nos lleva a reflexionar sobre cuestiones ampliamente discutidas del cotidiano, una vez que el equipo de enfermería es responsable de la preparación, almacenaje, aplazamiento y administración de las medicaciones, siendo una práctica que ocupa lugar preferente en la enfermería, asegurando con seguridad y eficacia el éxito del tratamiento del paciente ${ }^{(23)}$.

El equipo de enfermería actúa en la parte final del proceso de medicación, pudiendo prevenir los errores que por ventura acontezcan en otra etapa y siendo normalmente responsabilizada por este error ${ }^{(24)}$. Entre tanto, includo considerando el grado de afectación, responsabilidad y el dominio de la enfermería en el campo de los sistemas de medicación, encontramos solo un artículo ${ }^{(19)}$ escrito exclusivamente por enfermeras, que sumado a los otros artículos que en la relación de autores incluían eventualmente enfermeros, rindió un total de $13,3 \%$ de los autores.

La dificultad en tratar de forma clara los errores de medicación y sus consecuencias puede ser vista como un reflejo de la cultura occidental de abordaje del problema, donde el centro de la culpa recae sobre el individuo y no sobre el proceso. La característica punitiva de la gestión puede reducir sustancialmente la notificación de estos eventos y la creación de mecanismos de prevención basados en experiencias anteriores $^{(4-5,17,22)}$. Durante el análisis de los artículos recogidos, podemos observar que en uno de los artículos ${ }^{(19)}$, la palabra variances (en inglés) fue usada por las autoras para describir las discrepancias entre lo que era prescrito por el médico y lo que era administrado por el equipo de enfermería, denotando un abordaje más ameno y posiblemente más tímido de la problemática del error de administración de medicamentos.

El hecho de que solo tres artículos de investigaciones primarias ${ }^{(13,15,17)}$ se preocuparan en citar las consecuencias de los errores, corrobora la idea de que abordar los resultados y el impacto de los errores de medicamentos en la morbilidad y mortalidad de los recién nacidos internados en UTIN aún es un hecho velado y una laguna a ser completada. Resaltamos aún que de estos trabajos citados, ninguno 
objetivó directamente conocer la magnitud de las consecuencias de los errores de medicaciones y uno de ellos ${ }^{(17)}$ relata en los resultados que no hubo consecuencias (evento adverso).

Aunque la temática todavía está poco estudiada por la enfermería neonatal y la subnotificación evidente, un estudio brasileño ${ }^{(25)}$ midiendo el conocimiento sobre en qué consiste un error de medicación, su necesidad de notificación y el contenido de la misma, apuntó que $68 \%$ de los profesionales de enfermería entrevistados presentaron conocimientos sobre la definición de errores de medicación y 93\% afirmaron la necesidad de notificación, siendo que $85 \%$ poseen conocimiento sobre el contenido de la notificación.

Reconocer que los errores son frecuentes e identificar sus padrones y causas en el cotidiano de la práctica de atención al niño son apenas los primeros pasos para la reducción de los riesgos y aumento de la seguridad del paciente ${ }^{(4)}$.

En la categoría de análisis "ocurrencia", en relación a los errores en UTIN los autores fueron unánimes en concluir que la mayor parte de los errores ocurridos era de medicaciones. De estos, $60 \%$ estaban asociados a las medicaciones parenterales, en especial, a la administración de gentamicina ${ }^{(13)}$.

Las prácticas de terapia medicamentosa en la UTIN, debido a las características de la población, están enfocadas principalmente en la administración de medicamentos por vía intravenosa, comparativamente al adulto, que por otras vías más comúnmente utilizadas como la intramuscular y oral ${ }^{(26)}$. De esta forma, la ocurrencia mayor de errores en la terapia intravenosa se justifica por el hecho de ser una terapéutica esencial en el continuum de la atención neonatal y el equipo de enfermería posee una interface de destaque en ese contexto, asegurando con seguridad y eficacia el éxito del tratamiento del paciente ${ }^{(23)}$.

Los errores son clasificados de acuerdo con American Society of Hospital Pharmacists $(\mathrm{ASHP})^{(27)}$ en: error de prescripción, error de omisión, error de programación, error de medicación no autorizada, error de dosis, error en la preparación de medicaciones, error de técnica de administración, error de deterioro de drogas, error de monitoreo, error de adhesión y otros errores.

Según los autores, la ocurrencia de errores de medicación en la UTIN se dio principalmente por programación inadecuada de bombas de infusión ${ }^{(11)}$, errores de prescripción $^{(12)}$, errores de dosis ${ }^{(15-16)}$, errores de administración ${ }^{(16)}$ y errores de omisión ${ }^{(17)}$. Sin embargo, la mayoría de los errores eran evitables ${ }^{(15)}$.

Un estudio ${ }^{(28)}$ realizado en 21 Unidades de Cuidados Intensivos Pediátricos de São Paulo con una muestra de 110 enfermeros concluyó que la mayoría de las bombas de infusión utilizadas en las unidades no eran sometidas al mantenimiento preventivo. Ocurrencias adversas debidas a la bomba de infusión fueron identificadas por 95,5\% de los enfermeros.

El uso de bombas de infusión inteligentes puede prevenir la ocurrencia de errores en la administración de medicamentos, aumentando la seguridad del paciente. Sin embargo, debido a la falta de directrices para el uso de estos dispositivos y el alto costo de implantación, lleva a la conclusión de que aún pasará bastante tiempo hasta que esa tecnología sea incorporada en nuestro día a día ${ }^{(29)}$. 
Las categorías de análisis "factores relacionados" y "Mecanismos de prevención" fueron las que más incluyeron estudios del muestreo, evidenciando el carácter investigativo de los estudios en el sentido de correlacionar los mecanismos causales con las estrategias de prevención. Dentro de los factores relacionados a los errores de medicación en UTIN, los autores ${ }^{(12-13,19)}$ apuntaron principalmente la prescripción incompleta o ilegible y la falta de comunicación como uno de los principales factores asociados a los errores de medicación en UTIN. Asociado a este hecho, se estableció también la relación del error con la falta de uniformidad en los sistemas de medicaciones de la UTIN, incluyendo falta de medicamentos con formulaciones comerciales fraccionadas para la clientela neonatal, dosis que varían mucho de acuerdo con el peso, edad gestacional, edad corregida, y diferentes padrones de dosis para el mismo medicamento ${ }^{(14,18)}$.

Aproximadamente $80 \%$ de las drogas comercializadas para adultos no son destinadas para su uso en pediatría. Entre tanto, se observa cotidianamente, por la práctica clínica y estudios en el área, que la eficacia terapéutica de estos medicamentos está respaldada. Todavía no se observan mudanzas en la industria farmacéutica, a fin de que las formulaciones de estas drogas atiendan a las necesidades específicas del niño ${ }^{(26)}$.

Un estudio ${ }^{(30)}$ que objetivó identificar y analizar los puntos frágiles de la comunicación como una posible causa de errores de medicación, las prescripciones incompletas, con abreviaturas y realizadas sobre interrupciones y distracciones, las fallas en los relleno de formularios de dispensación y en la transcripción para etiquetas para preparación de medicaciones, así como la falta de comunicación entre profesionales pueden generar actos inseguros y configuran fuentes de errores de medicación.

Muchas veces, especialmente en unidades de tratamiento intensivo, los medicamentos prescritos son potencialmente peligrosos (MPP), pudiendo el error acarrear consecuencias todavía más graves. Otros autores ${ }^{(31)}$ encontraron una razón media de errores de prescripción de MPP de 3,3/prescripción, estando estos relacionados principalmente a la prescripción ilegible, siendo esa razón tres veces más frecuente cuando la prescripción es manuscrita en comparación a la prescripción pre-digitalizada.

En relación al horario de ocurrencia de los errores como posible factor relacionado, a pesar del intenso desgaste físico, mental y el riesgo para desatención en el horario de trabajo nocturno, no hubo consenso en la literatura analizada en relación a esa variable. Algunos autores ${ }^{(11)}$ concluyeron que la tasa de errores fue mayor durante el día, mientras otros ${ }^{(17)}$ encontraron tasas mayores durante la noche, y otro estudio ${ }^{(15)}$ no dio diferencia estadísticamente significativa entre las tasas de errores en los períodos del día estudiados.

El hecho de que buena parte de los artículos incluyeron los problemas con la prescripción y comunicación como posibles factores relacionados a los errores, es fortalecido en la categoría "Mecanismos de Prevención", donde de los 8 artículos incluidos en esa categoría de análisis, 6 citan la prescripción electrónica como una intervención con un alto impacto en la reducción de los errores de medicación en UTIN. De estos estudios, 4 objetivaron primariamente comparar en dos fases (pre y post-intervención) el impacto de la prescripción electrónica en la reducción de las tasas de errores y en todos ellos hubo diferencia estadísticamente significativa entre las variables analizadas ${ }^{(16,19-21)}$. 
Prescripciones electrónicas que incluyen recursos de apoyo a la decisión clínica ofrecen acceso inmediato a las informaciones del paciente, reducen la posibilidad de selección incorrecta del medicamento, garantizan prescripciones completas y formato adecuado, fortalecen soporte para cálculo y estandarización de dosis y volumen para reconstitución y diluciones, y proporcionan la posibilidad de verificar interacciones medicamentosas, contraindicaciones y alergia, pudiendo presentar sistemas de alerta en esas situaciones ${ }^{(4)}$.

La implementación de un sistema de gerenciamiento de riesgo con incorporación de una cultura de seguridad positiva, cambio en el abordaje del error y gestión no punitiva también fueron descritas como estrategias de prevención ${ }^{(17)}$.

Se enfatiza también que la combinación de programas educativos con la intención de contribuir a la mejora de las competencias y habilidades en la administración de medicamentos, la participación activa de farmacéuticos clínicos y el desarrollo de la cultura local, divulgando algoritmos claros y accesibles para orientar el comportamiento cuando hay ocurrencia de errores, son mecanismos que pueden minimizar la ocurrencia de errores de medicación en la UTIN ${ }^{(13,17)}$.

\section{CONSIDERACIONES FINALES}

Aunque la falta de más estudios robustos sobre el tema perjudique una evaluación más profunda, la presente revisión integrativa permitió un panorama general de las producciones científicas disponibles en las bases de datos sobre los errores de medicación en la UTIN, sus dimensiones en la atención de enfermería al recién nacido gravemente enfermo y complejidad envuelta en esta temática.

Los resultados apuntaron que la administración de medicamentos es una práctica destacada en la unidad neonatal y que la ocurrencia de errores es alta y frecuentemente asociada a los problemas de comunicación, prescripción y falta de estandarización de formulaciones y dosis para recién nacidos. El equipo de enfermería tiene una responsabilidad impar en este contexto, porque es quien actúa directa e indirectamente en todos los procesos del sistema de medicación. La responsabilidad ética y los avances tecnológicos imponen a los profesionales de enfermería la búsqueda constante de conocimientos específicos y actualizados.

Con la evolución cultural de la sociedad y la mayor notificación de los errores y eventos adversos, algunos factores relacionados y estrategias de prevención son descritos en la literatura para minimizar iatrogenias de la práctica medicamentosa. Por lo tanto, para la evolución de este sistema de notificación y vigilancia, es condición sine qua non el envolvimiento de todos los profesionales del equipo multidisciplinar y el cambio de la cultura de abordaje del error.

Errar es una condición intrínseca irreversible del ser humano. Entre tanto notamos que admitir y conocer la incidencia, los factores relacionados y las consecuencias de los errores, transformando la falla en una oportunidad de mejora, es el camino para la construcción de un sistema de salud más seguro y de calidad. 


\section{REFERENCIAS BIBLIOGRÁFICAS}

1- Guinsburg R. Avaliação e tratamento da dor no recém-nascido. J Pediatr [internet]. 1999 [acesso 16 abr 2012];75(3):149-160. Disponível em: http://www.jped.com.br/conteudo/99-75-03-149/port.pdf

2- Leite NS, Vieira M, Veber AP. Estudos de utilização de medicamentos: uma síntese de artigos publicados no Brasil e América Latina. Ciência \& Saúde Coletiva [internet]. 2008 [acesso 16 abr 2012];13 Suppl 739-802. Disponível em: http://www.scielo.br/pdf/csc/v13s0/a29v13s0.pdf

3- Rissalto MAR, Romano-Lieber NS, Lieber RR. Terminologia de acidentes com medicamentos no contexto hospitalar. Cad Saúde Pública [internet]. 2008 [acesso 16 abr 2012];24(9):1965-1975. Disponível em: http://www.scielo.br/pdf/csp/v24n9/02.pdf

4- Belela ASC, Pedreira MLG, Peterlini MAS. Erros de medicação em pediatria. Rev Bras Enferm [internet]. 2011 [acesso 16 abr 2012];64(3):563-9. Disponível em: http://www.scielo.br/pdf/reben/v64n3/v64n3a22.pdf

5- Kohn LT, Corrigan JM, Donaldson MS. Errors in the health care: a leading cause of death and injury. In: Kohn LT, Corrigan JM, Donaldson MS. To error is human: building a safer helth system. Washington DC: Committee on Quality of Health Care in America, National Academy of Instituite of Medicine; 2000. p. 26-48.

6- Pedreira ML, Marin HF. Patient safety initiatives in Brazil: a nursing perspective. Int J Med Inform. 2004;73(7-8):563-7.

7- Menezes Brito MJ, De Mattia Rocha A, Resende Ferreira V. Análisis de los eventos adversos en uma unidad de terapia intensiva neonatal como herramienta de gestión de calidad de la atención de enfermería. Enfermería Global [Internet]. 2009 [acesso 23 out 2012];8(3):1-13. Disponível em: http://revistas.um.es/eglobal/article/view/75161/72911

8- Mendes KDS, Silveira RCCP, Galvão CM. Revisão integrativa: método de pesquisa para a incorporação de evidências na saúde e na enfermagem. Texto Contexto Enferm [Internet]. 2008 [acesso 16 abr 2012];17(4):758-64. Disponível em: http://redalyc.uaemex.mx/redalyc/pdf/714/71411240017.pdf

9- Souza MT, Silva MD, Carvalho R. Revisão integrativa: o que é e como fazer. Einstein [internet]. 2010 [acesso 16 abr 2012];8(1):102-6. Disponível em: http://apps.einstein.br/revista/arquivos/PDF/1134-Einsteinv8n1_p102-106_port.pdf

10- Polit DF, Beck CT, Hungler BP. Study guide to accompany essentials of nursing research: methods, appraisals, and utilization. 5th.ed. Philadelphia: Lippincott; 2001c.

11- Lerner RB, Carvalho M, Vieira AA, Lopes JMA, Moreira MEL. Erros de medicação em unidades de terapia intensiva neonatal. J Pediatr (Rio J) [internet]. 2008 [acesso 16 abr 2012];84(2):166-170. Disponível em: http://www.scielo.br/pdf/jped/v84n2/v84n2a13.pdf

12- Snijders C, Van Lingen RA, Molendijk A, Fetter WPF. Incidents and errors in neonatal intensive care: a review of the literature. Arch Dis Child Fetal Neonatal [internet]. 2007 [acesso 16 abr 2012];92:391-398. Disponível em: http://portalneonatal.com.br/uti-neonatal/arquivos/errosemUTINN.pdf

13- Simpson JH, Lynch R, Grant J, Alroomi L. Reducing medication errors in the neonatal intensive care unit. Arch Dis Child Fetal Neonatal [internet]. 2004 [acesso 16 abr 2012];89:480-2. Disponível em: http://fn.bmj.com/content/89/6/F480.full.pdf

14- Gray JE, Goldmann DA. Medication errors in the neonatal intensive care unit: special patients, unique issues. Arch Dis Child Fetal Neonatal [internet]. 2004 [acesso 16 abr 2012];89:472-3. Disponível em: http://fn.bmj.com/content/89/6/F472.full.pdf 15- Jain S, Basu S, Parmar VR. Medication errors in neonates admitted in intensive care unit and emergency departament. Indian J Med Sci [internet]. 2009 [acesso 16 abr 2012];63:145-51. Disponível em: http://www.indianjmedsci.org/article.asp?issn=00195359; year $=2009 ;$ volume $=63$; issue $=4 ;$ spage $=145$; epage $=151$; aulast $=$ Jain 
16- Walsh KE, Landrigan CP, Adams WG, Vinci RJ, Chesare JB, Cooper MR, et al. Effect of computer order entry on prevention of serious medication errors in hospitalized children. Pediatrics [internet]. 2008 [acesso 16 abr 2012];121(3):421-42. Disponível em: http://pediatrics.aappublications.org/content/121/3/e421.full.pdf+html

17- Otero P, Leyton A, Mariani G, Cernadas JMC. Medication errors in pediatric inpatients: prevalence and result of a prevention program. Pediatrics [internet]. 2008 [acesso 16 abr 2012];122(2):737-43. Disponível em: http://pediatrics.aappublications.org/content/122/3/e737.full.pdf+html

18- Chappell K, Newman C. Potential tenfold drug overdoses on a neonatal unit. Arch Dis Child Fetal Neonatal [internet]. 2004 [acesso 16 abr 2012];89:483-484. Disponível em: http://fn.bmj.com/content/89/6/F483.full.pdf+html

19- Taylor JA, Loan LA, Kamara J, Blackburn S, Whitney D. Medication administration variances before and after implementation of computadorized physican order entry in a neonatal intensive care unit. Pediatrics [internet]. 2008 [acesso 16 abr 2012];121(1):123-128. Disponível em:

http://pediatrics.aappublications.org/content/121/1/123.full.pdf

20- Lehmann CU, Conner KG, Cox JM. Provider error prevention: online Total Parenteral Nutrition Calculator. Proc AMIA Symp [internet]. 2002 [acesso 16 abr 2012];435-9.

Disponível

em:

http://www.ncbi.nlm.nih.gov/pmc/articles/PMC2244250/pdf/procamiasymp00001-0476.pdf

21- Lehmann CU, Conner KG, Cox JM. Provider error prevention: online Total Parenteral Nutrition Calculator. Pediatrics [internet]. 2004 [acesso 16 abr 2012];113(4):748-53. Disponível em: http://pediatrics.aappublications.org/content/113/4/748.full.pdf+html

22- Oliveira RB, Melo ECP. O sistema de medicações de um hospital especializado no município do Rio de Janeiro. Esc Anna Nery [internet]. 2011 [acesso 16 abr 2012];15(3):480-489. Disponível em: http://www.scielo.br/pdf/ean/v15n3/a06v15n3.pdf 23- Pedreira MLG, Chaud MN. Terapia intravenosa em pediatria: subsídios para a prática da enfermagem. Acta Paul Enferm. 2004; 17(2):222-8.

24- Cassiani SHB, Teixeira TCA, Opitz SP, Linhares JC. O Sistema de medicações nos hospitais e sua avaliação por um grupo de profissionais. Rev Esc Enferm USP [internet]. 2005 [acesso 16 abr 2012];39(3):280-7. Disponível em: http://www.scielo.br/pdf/reeusp/v39n3/05.pdf

25- Telles PCP Filho, Praxedes MFS, Pinheiro MLP. Erros de medicação: análise do conhecimento da equipe de enfermagem de uma instituição hospitalar. Rev Gaúcha Enferm [internet]. 2011 [acesso 16 abr 2012];32(3):539-45. Disponível em: http://www.scielo.br/pdf/rgenf/v32n3/15.pdf

26- Peterlini MAS, Chaud MN, Pedreira MLG. Drug therapy orphans: the administration of intravenous drugs in hospitalized children. Rev. Latino-Am. Enfermagem [internet]. 2003 [acesso 16 abr 2012];11(1):88-95. Disponível em: http://www.scielo.br/pdf/rlae/v11n1/16564.pdf

27- American Society of Hospital Pharmacists - ASHP. Guideline on preventing medication errors in hospitals. Am J Hosp Pharm [internet]. 1993 [acesso 16 abr 2012];50:305-14. Disponível em: http://www.ashp.org/s_ashp/docs/files/MedMis_Gdl_Hosp.pdf

28- Pedreira, MLG. Uso de bombas de infusão na terapia intravenosa em crianças assistidas em unidades de cuidados intensivos pediátricos: contribuições para estudos clínicos e técnicos. [Tese] [internet] São Paulo: UNIFESP-EPM; 1999.

29- Pedreira MLG, Peterlini MAS, Harada MJCS. Tecnologia da informação e prevenção de erros de medicação em pediatria: prescrição informatizada, código de barras e bombas de infusão inteligentes. Rev Soc Bras Enferm Ped [internet]. 2005 $\begin{array}{llllll}\text { [acesso } & 16 & \text { 2012];5(1):55 Disponível em: }\end{array}$ 
http://www.sobep.org.br/revista/images/stories/pdf-revista/vol5-n1/v.5_n.1-art7.revitecnologia-da-informacao-e-prevencao-de-erro.pdf

30- Silva AEBC, Cassiani SHB, Miasso AI, Optiz SP. Problemas na comunicação: uma possível causa de erros de medicação. Acta Paul Enferm [Internet]. 2007 [acesso 16 abr 2012];20(3):272-6. Disponível em: http://www.scielo.br/pdf/ape/v20n3/a05v20n3.pdf

31- Rosa MB, Perini E, Anacleto TA, Neiva HM, Bogutchi T. Erros na Prescrição hospitalar de medicamentos potencialmente perigosos. Rev Saúde Pública [internet]. 2009 [acesso 16 abr 2012];43(3):490-8. Disponível em: http://www.scielo.br/pdf/rsp/v43n3/7265.pdf 